\title{
ATLAS probe for the study of galaxy evolution with $300,000,000$ galaxy spectra
}

Robert Content, Yun Wang, Massimo Roberto, Mark Dickinson, Henry Ferguson, et al.

Robert Content, Yun Wang, Massimo Roberto, Mark Dickinson, Henry Ferguson, Lynne Hillenbrand, Wesley Fraser, Peter Behroozi, Jarle Brinchmann, Andrea Cimatti, Emanuele Daddi, Christopher Hirata, Michael Hudson, J. Davy Kirkpatrick, Robert Barkhouser, James Bartlett, Robert Benjamin, Ranga Chary, Charlie Conroy, Megan Donahue, Olivier Doré, Peter Eisenhardt, Karl Glazebrook, George Helou, Sangeeta Malhotra, Lauro Moscardini, Zoran Ninkov, Alvaro Orsi, Michael Ressler, James Rhoads, Jason Rhodes, Alice Shapley, Stephen Smee, "ATLAS probe for the study of galaxy evolution with $300,000,000$ galaxy spectra," Proc. SPIE 10698, Space Telescopes and Instrumentation 2018: Optical, Infrared, and Millimeter Wave, 106980I (22 August 2018); doi: 10.1117/12.2313082

Event: SPIE Astronomical Telescopes + Instrumentation, 2018, Austin, Texas, United States 


\title{
ATLAS Probe for the study of galaxy evolution with 300,000,000 galaxy spectra
}

Robert Content*a $^{* \mathrm{a}}$, Yun Wang ${ }^{\mathrm{b}}$, Massimo Roberto ${ }^{\mathrm{c}, \mathrm{d}}$, Mark Dickinson ${ }^{\mathrm{e}}$, Henry Ferguson ${ }^{\mathrm{c}}$, Lynne Hillenbrand $^{\mathrm{b}}$, Wesley Fraser ${ }^{\mathrm{g}}$, Peter Behroozi ${ }^{\mathrm{r}}$, Jarle Brinchmann ${ }^{\mathrm{k}}$, Andrea Cimatti, ${ }^{1, \mathrm{~s}}$, Emanuele Daddy $^{\mathrm{n}}$, Christopher Hirata ${ }^{\mathrm{f}}$, Michael Hudson ${ }^{\mathrm{t}}$, J. Davy Kirkpatrick ${ }^{\mathrm{b}}$, Robert Barkhouser ${ }^{\mathrm{d}}$, James Bartlett $^{\mathrm{h}}$, Robert Benjamin ${ }^{\mathrm{i}}$, Ranga Chary ${ }^{\mathrm{b}}$, Charlie Conroy ${ }^{\mathrm{m}}$, Megan Donahue ${ }^{\mathrm{o}}$, Olivier Doré ${ }^{\mathrm{h}}$, Peter Eisenhardt $^{\mathrm{h}}$, Karl Glazebrook ${ }^{\mathrm{u}}$, George Helou ${ }^{\mathrm{b}}$, Sangeeta Malhotra ${ }^{\mathrm{p}}$, Lauro Moscardinil ${ }^{\mathrm{l}, \mathrm{v}, \mathrm{w}}$, Zoran Ninkov $^{\mathrm{e}}$, Alvaro Orsi ${ }^{\mathrm{x}}$, Michael Ressler ${ }^{\mathrm{h}}$, James Rhoads ${ }^{\mathrm{p}}$, Jason Rhodes ${ }^{\mathrm{h}}$, Alice Shapley ${ }^{\mathrm{q}}$, Stephen Smee $^{\mathrm{d}}$

${ }^{\mathrm{a}}$ Australian Astronomical Optics, 105 Delhi Road, North Ryde, NSW 2113, Australia; ${ }^{\mathrm{b}}$ IPAC, California Institute of Technology, Mail Code 314-6, 1200 E. California Blvd., Pasadena, CA 91125, USA; 'Space Telescope Science Institute, 3700 San Martin Drive, Baltimore, MD 21218, USA;

${ }^{\mathrm{d}}$ Dept. of Physics \& Astronomy, Johns Hopkins Univ., 3400 N. Charles Street, Baltimore, MD 21218, USA; ' National Optical Astronomy Observatory, 950 North Cherry Ave., Tucson, AZ 85719, USA; ${ }^{\mathrm{f}}$ Center for Cosmology and Astroparticle Physics, The Ohio State Univ., 191 West Woodruff Avenue, Columbus, OH 43210, USA; ${ }^{\mathrm{g} S}$ School of Mathematics and Physics, Queen's Univ. Belfast, University Road, Belfast, BT7 1NN, Northern Ireland, UK; ${ }^{\mathrm{h}} \mathrm{Jet}$ Propulsion Lab., California Institute of Technology, 4800 Oak Grove Drive Pasadena, CA 91011, USA; ${ }^{\mathrm{i}}$ Dept. of Physics, University of

Wisconsin - Whitewater, 800 W. Main Street Whitewater, WI 53190-1790, USA; ${ }^{\mathrm{k}}$ Leiden

Observatory, Leiden Univ., P.O. Box 9513, NL-2300 RA, Leiden, Netherland; ${ }^{\text {Department of }}$

Physics and Astronomy, Alma Mater Studiorum University of Bologna, via Gobetti 93/2, I-40129 Bologna, Italy; ${ }^{\mathrm{m}}$ Harvard-Smithsonian Ctr. for Astrophysics, 60 Garden St, Cambridge, MA 02138,

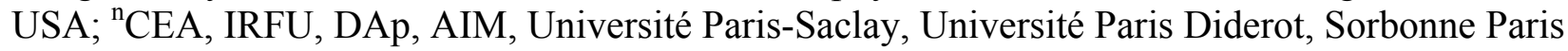

Cité, CNRS, F-91191 Gif-sur-Yvette, France; ${ }^{\circ}$ Physics and Astronomy Dept., Michigan State University, 567 Wilson Rd., East Lansing, MI 48824, USA; ${ }^{\mathrm{p}}$ NASA Goddard Space Flight Ctr., 8800 Greenbelt Rd, Greenbelt, MD 20771, USA; ${ }^{\mathrm{q}}$ Dept. of Physics \& Astronomy, UCLA, 430 Portola Plaza, Box 951547, Los Angeles, CA 90095-1547, USA; ' Steward Observatory, University of Arizona, 933 N Cherry Ave, Tucson, AZ 85719; ${ }^{\mathrm{s}} 11 \mathrm{INAF}$ - Osservatorio Astrofisico di Arcetri, Largo E. Fermi 5, I-50125, Firenze, Italy; ${ }^{\mathrm{t} D e p t}$. of Physics \& Astronomy, University of Waterloo,

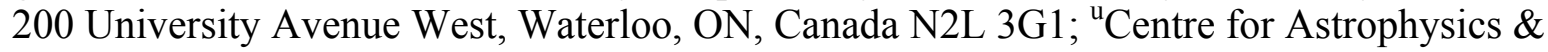
Supercomputing, Mail number H29, Swinburne University of Technology, PO Box 218, Hawthorn, Victoria 3122, Australia; ${ }^{\mathrm{V} I N A F}$ - Osservatorio di Astrofisica e Scienza dello Spazio di Bologna, via Gobetti 93/3, I-40129 Bologna, Italy; ${ }^{\mathrm{w}}$ INFN - Sezione di Bologna, viale Berti Pichat 6/2, I-40127 Bologna, Italy; ${ }^{\mathrm{x}}$ Centro de Estudios de Física del Cosmos de Aragón, Plaza de San Juan 1, Teruel, 44001, Spain

\begin{abstract}
ATLAS (Astrophysics Telescope for Large Area Spectroscopy) Probe is a mission concept for a NASA probe-class space mission with primary science goal the definitive study of galaxy evolution through the capture of $300,000,000$ galaxy spectra up to $z=7$. It is made of a $1.5-\mathrm{m}$ Ritchey-Chretien telescope with a field of view of solid angle $0.4 \mathrm{deg}^{2}$.
\end{abstract}

*robert.content@aao.gov.au; phone 61-2-9372-4846

Space Telescopes and Instrumentation 2018: Optical, Infrared, and Millimeter Wave, edited by Makenzie Lystrup, Howard A. MacEwen, Giovanni G. Fazio, Proc. of SPIE Vol. 10698, 106980I (c) 2018 SPIE · CCC code: 0277-786X/18/\$18 - doi: 10.1117/12.2313082 
The wavelength range is at least $1 \mu \mathrm{m}$ to $4 \mu \mathrm{m}$ with a goal of $0.9 \mu \mathrm{m}$ to $5 \mu \mathrm{m}$. Average resolution is 600 but with a possible trade-off to get 1000 at the longer wavelengths. The ATLAS Probe instrument is made of 4 identical spectrographs each using a Digital Micro-mirror Device (DMD) as a multi-object mask. It builds on the work done for the ESA SPACE and Phase-A EUCLID projects. Three-mirror fore-optics re-image each sub-field on its DMD which has 2048 x 1080 mirrors $13.6 \mu \mathrm{m}$ wide with 2 possible tilts, one sending light to the spectrograph, the other to a light dump. The ATLAS Probe spectrographs use prisms as dispersive elements because of their higher and more uniform transmission, their larger bandwidth, and the ability to control the resolution slope with the choice of glasses. Each spectrograph has 2 cameras. While the collimator is made of 4 mirrors, each camera is made of only one mirror which reduces the total number of optics. All mirrors are aspheric but with a relatively small P-V with respect to their best fit sphere making them easily manufacturable. For imaging, a simple mirror to replace the prism is not an option because the aberrations are globally corrected by the collimator and camera together which gives large aberrations when the mirror is inserted. An achromatic grism is used instead. There are many variations of the design that permit very different packaging of the optics. ATLAS Probe will enable ground-breaking science in all areas of astrophysics. It will (1) revolutionize galaxy evolution studies by tracing the relation between galaxies and dark matter from the local group to cosmic voids and filaments, from the epoch of reionization through the peak era of galaxy assembly; (2) open a new window into the dark universe by mapping the dark matter filaments to unveil the nature of the dark Universe using 3D weak lensing with spectroscopic redshifts, and obtaining definitive measurements of dark energy and modification of gravity using cosmic large-scale structure; (3) probe the Milky Way's dust-shrouded regions, reaching the far side of our Galaxy; and (4) characterize asteroids and other objects in the outer solar systems.

Keywords: Space telescope, NIR spectroscopy, Digital Micro-mirror Devices, prism spectroscopy, Multi-Object Spectroscopy.

\section{INTRODUCTION}

The observational data from recent years have greatly improved our understanding of the Universe. Some fundamental questions that remain to be studied in the coming decades include:

(1a) How have galaxies evolved? What is the origin of the diversity of galaxies?

(1b) What is the dark matter that dominates the matter content of the Universe?

(2) What is the dark energy that is driving the accelerated expansion of the Universe?

(3) What is the 3D structure and stellar content in the dust-enshrouded regions of the Milky Way?

(4) What is the census of objects in the outer Solar System?

The upcoming space missions Euclid ${ }^{1}$, WFIRST ${ }^{2}$, and JWST, and ground-based projects like LSST $^{3}$ will help us make progress in these areas through the synergy of imaging and spectroscopic data. In particular, Euclid, WFIRST, and LSST are complementary to each other, and jointly form a strong program for probing the nature of dark energy. However, in the case of massive spectroscopic surveys on Euclid and WFIRST, the instrumental designs of these projects limit their capabilities to fully address the fundamental questions described above. Both Euclid and WFIRST employ slitless grism spectroscopy, which increases background noise and will limit their capability to probe galaxy evolution science. Euclid and WFIRST spectra cover limited wavelength ranges, severely restricting opportunities to measure multiple diagnostic emission lines, and their red wavelength limits at $1.85 \mu \mathrm{m}$ and $1.95 \mu \mathrm{m}$, respectively, limiting the redshift range over which emission lines can be detected. JWST has slit spectroscopic capability, but a relatively small field of view (FoV), thus will not be suitable for carrying out surveys large enough to probe the relation between galaxy evolution and galaxy environment in a statistically robust manner. LSST has no spectroscopic capability, works only in the visible, and is limited by Earth's atmosphere. The lack of slit spectroscopy from space over a wide FoV is the obvious gap in current and planned future space missions ${ }^{4}$.

In order to obtain definite data sets to study galaxy evolution in diverse environments and within the cosmological context, we need spectroscopy with high multiplicity and low background noise from space. In this paper, we present the mission concept for ATLAS (Astrophysics Telescope for Large Area Spectroscopy) Probe, a $1.5 \mathrm{~m}$ space telescope with a FoV of $0.4 \mathrm{deg}^{2}$, a spectral resolution of $\mathrm{R}=600$ over the wavelength range from $1 \mu \mathrm{m}$ to $4 \mu \mathrm{m}$, and an unprecedented 
spectroscopic capability based on Digital Micro-mirror Devices (DMDs) with spectroscopic multiplex as high as 5,000 to 10,000 targets per observation.

We present a preliminary design for the ATLAS instrument. It is based on a design first developed for the SPectroscopic All-sky Cosmic Explorer (SPACE) proposal, then for the first version of EUCLID. At the time, 2 space telescope proposals for the study of dark energy were submitted to answer the call for proposals of the ESA Cosmic Vision 20152025. One was SPACE, the other the Dark UNiverse Explorer (DUNE). The payload of DUNE was made of 2 cameras, one for the visible to measure galaxy weak lensing, the other in the NIR to measure their photometric redshifts. SPACE was the precursor of ATLAS Probe. Its payload was a spectrograph with DMDs used as addressable masks covering the same field of view than ATLAS Probe. The telescope and slit sizes were also the same. The wavelength range was however much smaller and limited to the NIR: $0.9 \mu \mathrm{m}$ to $1.7 \mu \mathrm{m}$ instead of $1 \mu \mathrm{m}$ to $4 \mu \mathrm{m}$ for ATLAS Probe. Its main goal was the measurement of the Baryonic Acoustic Oscillations (BAO). However, ESA did not choose one mission or the other or both but pre-selected both proposals for one dark energy mission. A pre-phase-A study was then necessary to clarify this situation. The 3 choices were a) keep SPACE and drop DUNE, b) keep DUNE and drop SPACE, and c) merge the 2 missions. The latter was the preferred solution of ESA which sees in this option a minimum multiplex advantage of 2, possibly 4, compared to the option of keeping one of the 2 missions. The assessment study resulted in a merged mission called EUCLID. The study led to a possible concept for the mission and the payload ${ }^{5}$ developed by the first author of the present paper, paving the way for the industrial studies. The SPACE payload became the EUCLID spectrograph channel. The DUNE payload became the EUCLID imaging channel itself split between the EUCLID visible channel and the EUCLID NIR photometric channel.

Table 1. ATLAS Probe main characteristics.

\begin{tabular}{|c|c|c|}
\hline Parameter & & Notes \\
\hline Aperture & $1.5 \mathrm{~m}$ & Probe-class mission, can launch in 2030 \\
\hline Field of View & 0.4 deg $^{2}$ & Slitsize $0.75^{\prime \prime} \times 0.75^{\prime \prime}$ \\
\hline Wavelength range & $1-4 \mu \mathrm{m}$ & Near and mid IR \\
\hline Spectral Resolution & $\mathrm{R} \sim 600$ & Heritage of mature designs \\
\hline Multiplex factor & $5,000-10,000$ & Uses DMDs, $\sim 9,000,000$ micro-mirrors \\
\hline \# of galaxy spectra & $300 \mathrm{M}$ in $4 \mathrm{yrs}$ & $\begin{array}{l}\text { 3-tiered galaxy surveys, } \mathrm{AB} \text { mag }=24.0 \text {, } \\
25.5,26.8(3 \sigma)\end{array}$ \\
\hline Redshift range & $0.5<z<7$ & Emission line and passive galaxies \\
\hline Estimated cost & $\$ 1 \mathrm{~B}$ & DMDs can reach TRL 6 within two years \\
\hline
\end{tabular}

ESA requested that the number of optics in the SPACE instrument be significantly reduced for the EUCLID spectrograph channel. This led to a reduction of the number of optics before the DMDs and in the cameras of the spectrographs, and a reduction in the number of arms. The resulting design of the DMD fore-optics and the spectrographs are the basis of the present design of the ATLAS Probe payload. In the phase-A, however, ESA abandoned the SPACE concept and replaced it by a much lower cost slitless spectroscopy system using grisms that can be inserted in the NIR camera of DUNE.

To understand the design of ATLAS Probe, it is necessary to first look at the design of SPACE and of the spectrograph channel of the original EUCLID. After a brief description of the ATLAS Probe science, we will present a description of these 2 designs, then the description of ATLAS Probe.

\section{ATLAS PROBE SCIENCE}

A complete description of the science case of ATLAS Probe can be found in reference 6 . Here we will highlight some of the exceptional science that ATLAS Probe will do.

ATLAS Probe is designed to provide the key data sets to address the fundamental questions listed at the beginning of Section 1. It is a concept for a NASA probe-class space mission for groundbreaking science in galaxy evolution, 
cosmology, and Milky Way studies. ATLAS Probe is the follow-up space mission to WFIRST that leverages WFIRST imaging for targeted spectroscopy, and multiplexes the scientific return of WFIRST by obtaining spectra of $\sim 90 \%$ of all galaxies imaged by the $\sim 2200 \mathrm{deg}^{2}$ WFIRST High Latitude Survey at $\mathrm{z}>0.5$.

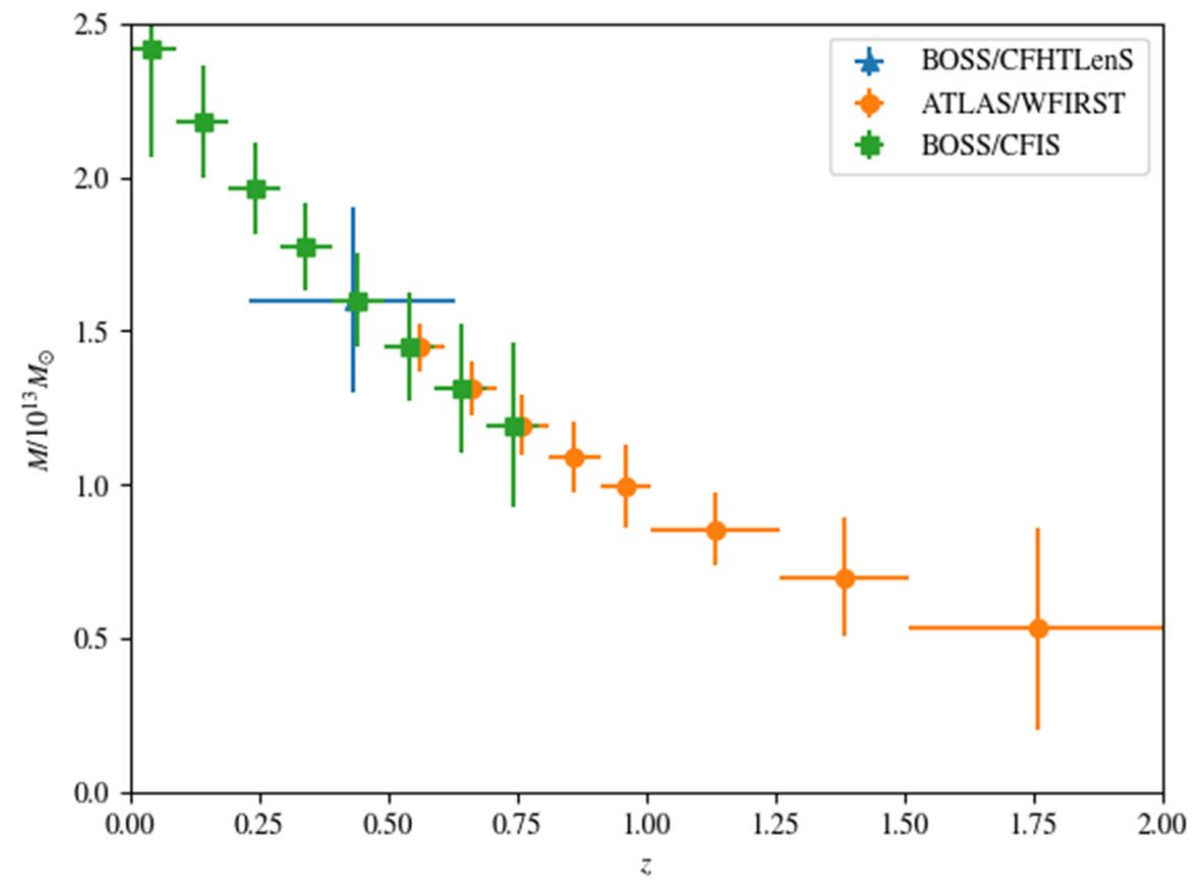

Figure 1. Conservative estimate of the measurement errors of filament mass from ATLAS Wide, for a 6-10 Mpc/h cosmic web filament between $10^{13} M_{\odot}$ halos.

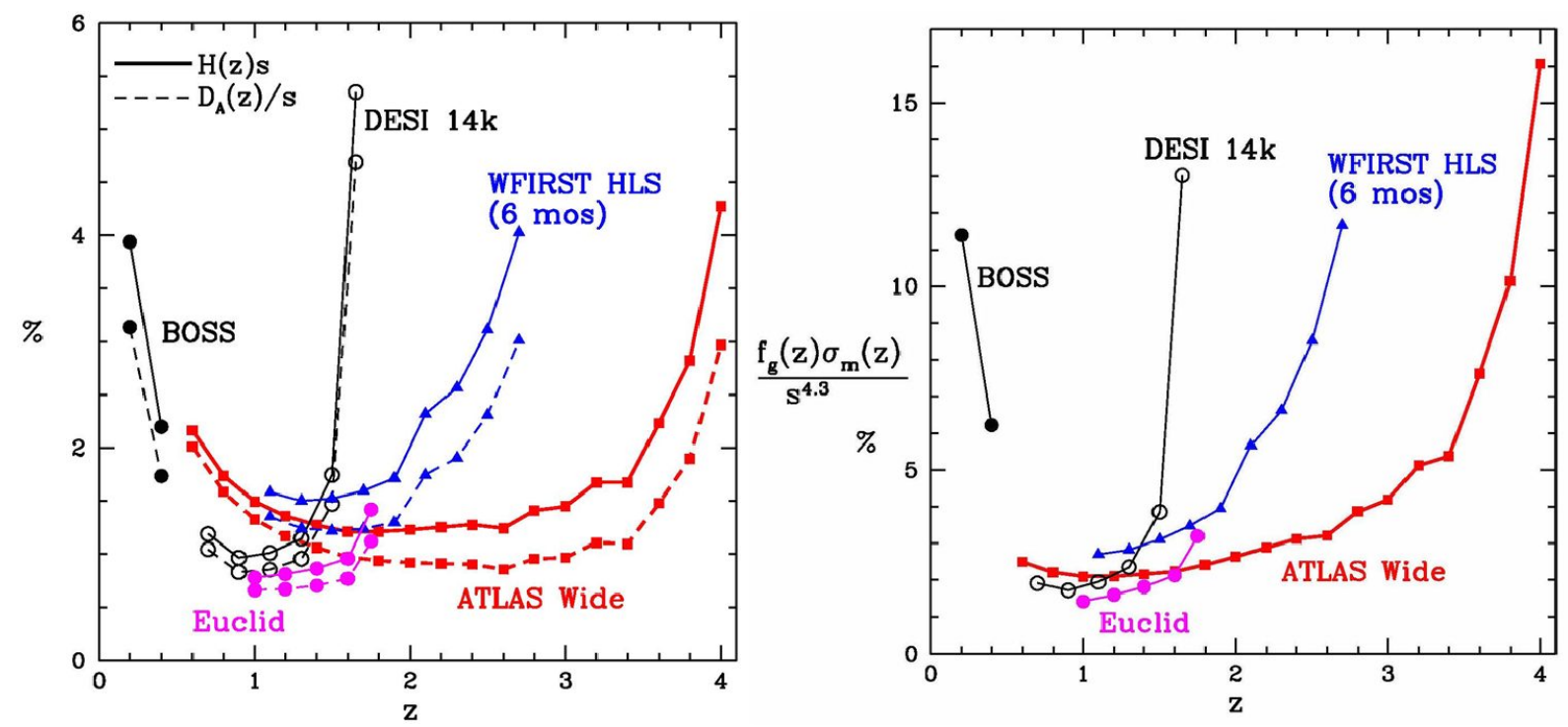

Figure 2. ATLAS vs. WFIRST and Euclid comparison of the measurement errors from BAO/RSD on the cosmic expansion history $H(\mathrm{z})$, angular diameter distance $D_{A}(\mathrm{z})$, and the growth rate of cosmic large-scale structure $f_{g}(\mathrm{z})$. Note that $s$ is the BAO scale, $\sigma_{m}(\mathrm{z}) \equiv G(\mathrm{z}) \tilde{P}_{0}^{1 / 2}$, where $G(\mathrm{z})$ is the growth factor, and $\tilde{P}_{0} \equiv P_{0} /\left[(\mathrm{Mpc} / h)^{3}(\mathrm{Mpc})^{\mathrm{ns}}\right]$, with $P_{0}$ denoting the power spectrum normalization (Wang, Chuang, \& Hirata 2013). The 14,000 deg ${ }^{2}$ ELG survey from the ground-based project DESI (Aghamousa et al. 2016) is also shown for comparison. 
ATLAS Probe science spans four broad categories that address the four fundamental questions:

(1) Revolutionize galaxy evolution studies by tracing the relation between galaxies and dark matter from galaxy groups to cosmic voids and filaments, from the epoch of reionization through the peak era of galaxy assembly. It will trace the relation between galaxies and dark matter with less than $10 \%$ shot noise on relevant scales at $1<\mathrm{z}<6$.

(2) Open a new window into the dark Universe by weighing the dark matter filaments in the cosmic web using 3D weak lensing, and obtaining definitive measurements of dark energy and tests of General Relativity using galaxy clustering. It will measure the mass of dark-matter-dominated filaments on the scales of $\sim 5-50 \mathrm{Mpch}^{-1}$ over the $2,200 \mathrm{deg}^{2}$ of WFIRST at $0.5<\mathrm{z}<3$.

(3) Probe the Milky Way's dust-enshrouded regions, reaching the far side of our Galaxy to a distance of $25 \mathrm{kpc}$.

(4) Characterize Kuiper Belt Objects and other planetesimals in the outer Solar Systems to probe its formation history through the composition of 3,000 comets and asteroids.
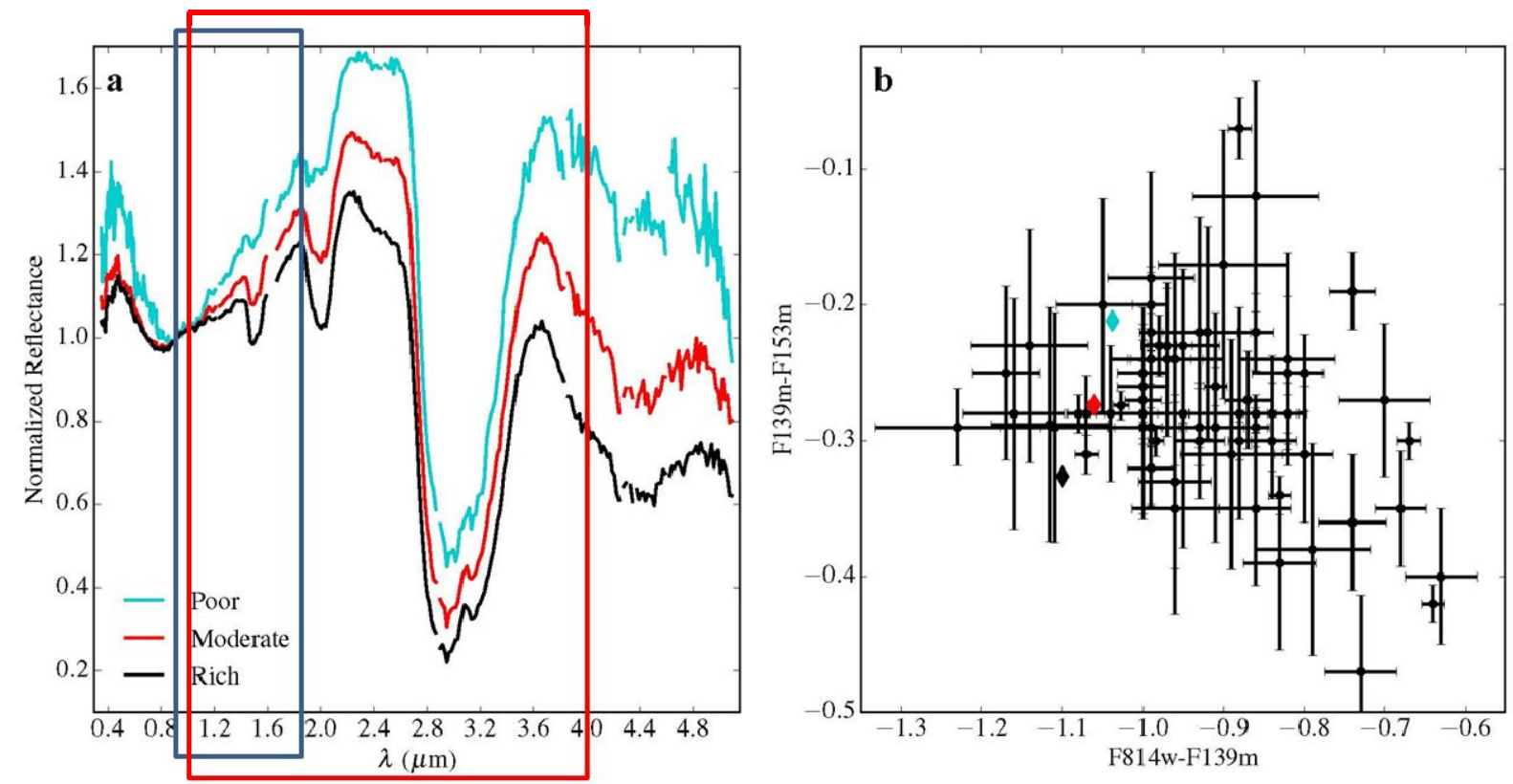

Figure 3. a: Spectra of the different spectra units found on Phoebe's surface. These spectra will appear in Fraser \& Brown (submitted). "Poor", "Moderate", and "Rich" represent different levels in the water absorption depth. In our observable KBO density estimates, we adopt the moderate spectrum which exhibits water-ice absorption typical of most KBOs. The blue rectangle shows the EUCLID spectral range while the red is ATLAS Probe's. b: NIR colors of KBOs gathered with the Wide Field Camera 3 on the Hubble Space Telescope (black points; see reference 9). Simulated colors of Phoebe of the spectra presented in panel a are shown by the colored points. The red point is that drawn from the moderate spectrum.

During its 5 year prime mission, ATLAS Probe will carry out three galaxy redshift surveys at high latitude (ATLAS Wide over $2200 \mathrm{deg}^{2}$, ATLAS Medium over $100 \mathrm{deg}^{2}$, and ATLAS Deep over $1 \mathrm{deg}^{2}$ ), a Galactic plane survey over 700 $\mathrm{deg}^{2}$, a survey of the outer Solar System over 1,200 $\mathrm{deg}^{2}$, as well as a Guest Observer program.

The galaxy evolution science objective provides the driving requirements for ATLAS Probe, which flow down to its spectral coverage of $1-4 \mu \mathrm{m}$; the requirements from the other science objectives are then easily met. It is well known that this spectral range represents a major advantage for galaxy evolution studies because of: (1) better sensitivity to stellar mass than rest-frame UV selection; (2) smaller K-corrections (i.e. sensitivity to all galaxy types, from star-forming to passive); (3) less affected by dust extinction; (4) coverage of the strongest optical rest frame lines (H6563 and [OIII]4959,5007) and those most useful for the physics of galaxy evolution. ATLAS Wide will use the WFIRST weak lensing imaging sample as the target list. ATLAS Medium and ATLAS Deep target lists will be magnitude selected from 
the lowest background regions of the WFIRST High Latitude Survey imaging, and/or from deeper WFIRST Guest Observer imaging programs.

In addition to definitive studies in galaxy evolution, ATLAS Wide Survey will enable ground-breaking studies in cosmology. ATLAS Galactic Plane Survey will explore dusty regions toward the Galactic center, and ATLAS Solar System Survey will probe the formation of the outer Solar System.

\section{ORIGINAL DESIGN OF SPACE}

In the original proposal of SPACE, the optical train can be separated into 3 subsystems: the telescope, the fore-optics that re-image the telescope focal plane onto 4 Digital Micromirror Devices (DMDs), and the spectrographs. The spectrographs themselves can be separated into 3 sub-systems: the collimators, the dispersive/filtering elements, and the cameras. This remains true for the design of the pre-phase-A EUCLID spectrograph channel but many of the characteristics are different. A complete description of the SPACE proposal, especially the science drivers, can be found in reference 4.

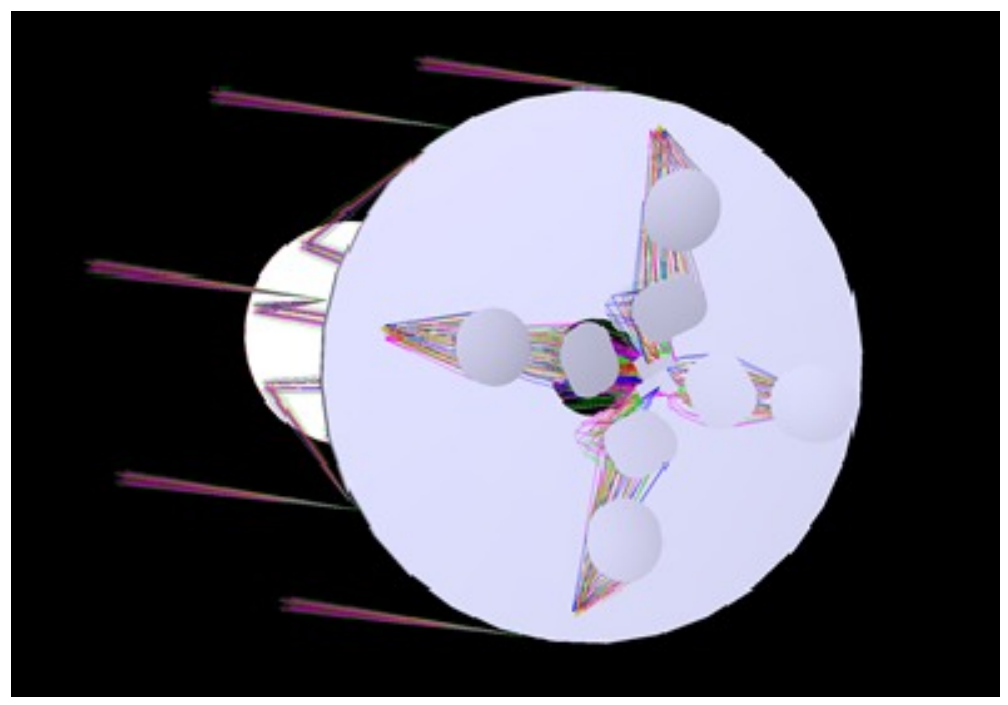

Figure 4. Telescope, fore-optics and DMDs of the SPACE proposal.

The telescope of the SPACE proposal is a Richer-Chrétien F/5.83. The primary is F/2.7 and $1.5-\mathrm{m}$ in diameter. In its focal plane, a pyramid mirror cuts the field into 4 subfields (Fig. 4) of $25.5^{\prime}$ x $13.5^{\prime}$ (65 $\mathrm{mm} \times 34 \mathrm{~mm}$ ) for a total coverage of almost $0.4 \mathrm{deg}^{2}$. Each subfield beam is reflected toward an optical system of 4 mirrors (hereafter referred to as the fore-optics) that images the subfield onto a DMD and adjusts its demagnification to fit the DMD size. The objects of interest in the field are reflected in a direction approximately normal to the DMD by turning the appropriate mirrors on the DMD to their 'ON' position while the rest of the field is reflected at an oblique angle towards a beam dump. Each DMD feeds a spectrograph. The 4-mirror collimator of each spectrograph delivers a pupil to a prism system in the dispersion mode, and a filter system in the imaging mode. The 4-lenses camera of each spectrograph delivers the image on its detector. The fore-optics and collimators are contained in a layer $460 \mathrm{~mm}$ deep behind the primary that takes advantage of the presence of the primary mirror support structure to reduce the total weight of the structure that holds the optics. The dispersing elements and the cameras are in a layer $160 \mathrm{~mm}$ deep just behind the fore-optics and collimator layer.

\subsection{Fore-optics and DMDs}

Each arm of the fore-optics re-images one of the 4 subfields onto one of 4 DMDs (figs. $4 \& 5$ ). They form a critical part of the system because of the unusual characteristics of the DMDs. Each DMD is made of an array of $2048 \times 1080$ micromirrors, $13.6 \mu \mathrm{m}$ on a side, which tilts along one of their diagonals by $+/-12^{\circ}$ (individually addressed ON and OFF 
positions). Each micro-mirror covers a square 0.75 " field that is imaged onto $2 \times 2$ pixels on the detector. To separate the incident and reflected "ON" beam, and get the reflected beam in a direction that is the most convenient for the design of the spectrograph, the beam axis must hits the DMD at an incident angle of $27^{\circ}$. Because of this incident angle, the field appears smaller in one direction than in the other so that the demagnified image must be smaller in that direction than in the other. The steep incident angle also makes it necessary to use off-axis optics. This favours the use of mirrors instead of lenses. The resulting beam is also relatively fast and elliptical at an average over the field of F/2.22 x F/2.49. The edges of the output geometric beam delivered to the spectrograph are included in an envelope of $F / 2.15 \times \mathrm{F} / 2.4$. The field edges must be accurately superimposed to the DMD edges to avoid losses and the PSF size should in principle be about 0.2 " or better to take advantage of the detector pixel size of 0.375 ". However, a point source must give a PSF that sufficiently covers the whole DMD micromirror to give a proper sampling on the detector. A very good image quality may then not be the best solution.
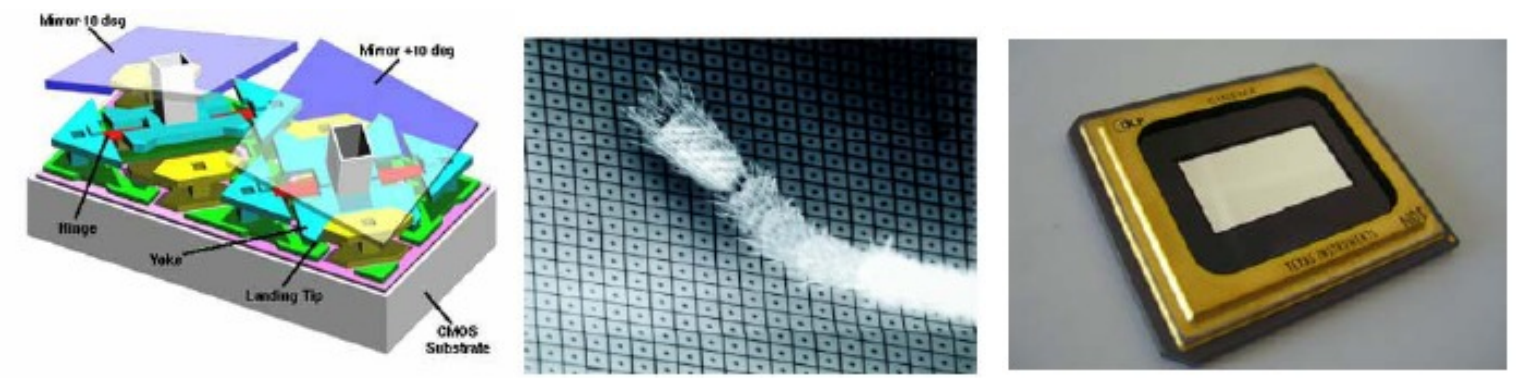

Figure 5. Typical substructure of a TI DMD (left); DMD array with an ant leg for comparison (centre); DMD CINEMA (right).

All of this is successfully performed by a system of 4 mirrors per arm (fig. 4) which then gives a high transmission as a bonus. The average PSF size (50\%EED) is 0.19 ". The smallest of the 4 re-imaging mirrors being near a pupil plane, it can be used as a cold stop. Only small modifications of the fore-optics are necessary with negligible effects on the image quality.

\subsection{Spectrographs}

Each DMD is followed by a prism spectrograph.

\subsubsection{Collimator}

The collimator is a 4 mirror optical system that re-images the DMD field at infinity (fig. 6). It also re-images on the prism the pupil with the size needed by the camera. The needed number of mirrors reflects the difficulty to have at the same time a fast off-axis input beam, a compact design, a collimated beam at the pupil image, a pupil away from any crossing beam so that the prism would not block some light, and a high image quality. The present design delivers an average PSF size of 0.13 ". It has little distortion which helps to avoid losses of field or detector surface at the edge of the detector and makes it easier to avoid cross-talks between spectra. This design was kept for EUCLID with some minor modifications.

\subsubsection{Dispersive element and filters}

The dispersive element is a prism. It is followed by a fold mirror that directs the beam in a plane perpendicular to the telescope optical axis (fig. 7). This permits to place the cameras in a layer just behind the collimators and fore-optics layer minimizing the overall space envelope. The main advantage here of a prism as dispersive element is that it can image a spectrum longer than one octave without order superposition. The present design permits a range of wavelengths from $0.6 \mu \mathrm{m}$ to $1.8 \mu \mathrm{m}$ simultaneously on the detector. The glass was chosen first for its low absorption in the wavelength range but also to deliver a spectral resolution as uniform as possible to fit the model used for the science simulations. The simulations were done with a constant resolution of 400 from $0.8 \mu \mathrm{m}$ to $1.8 \mu \mathrm{m}$, not with a constant element of resolution as would be given by a grating. In the final design, the glass would have been chosen to have an 
average slope of resolution versus wavelength that maximizes the scientific return. The variations in spectral length over the detector are only of a few percent so the resolution is also quite uniform between spatial positions on the DMD.

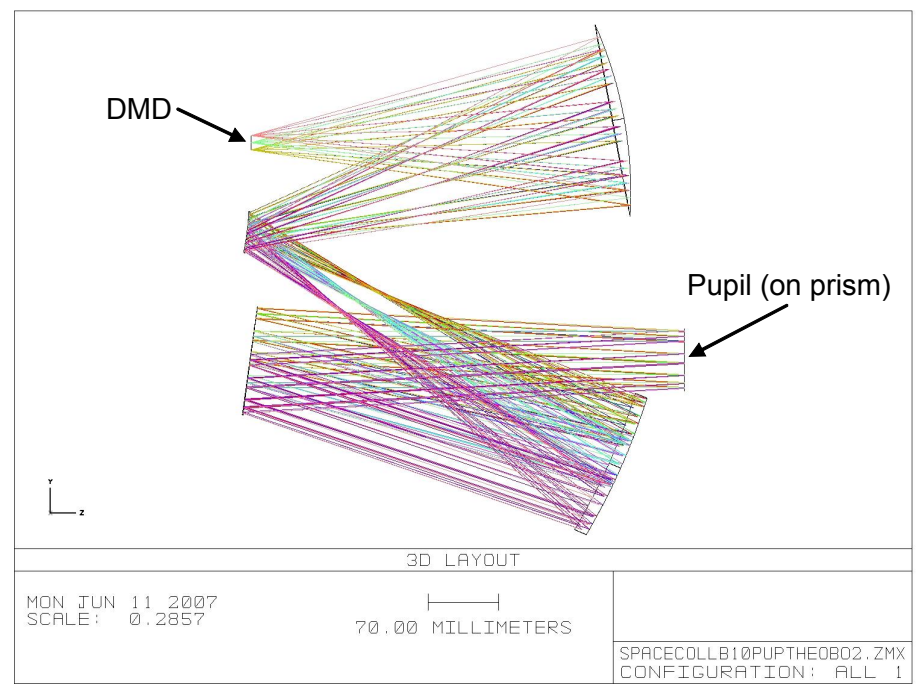

Figure 6. Layout of the SPACE collimator.

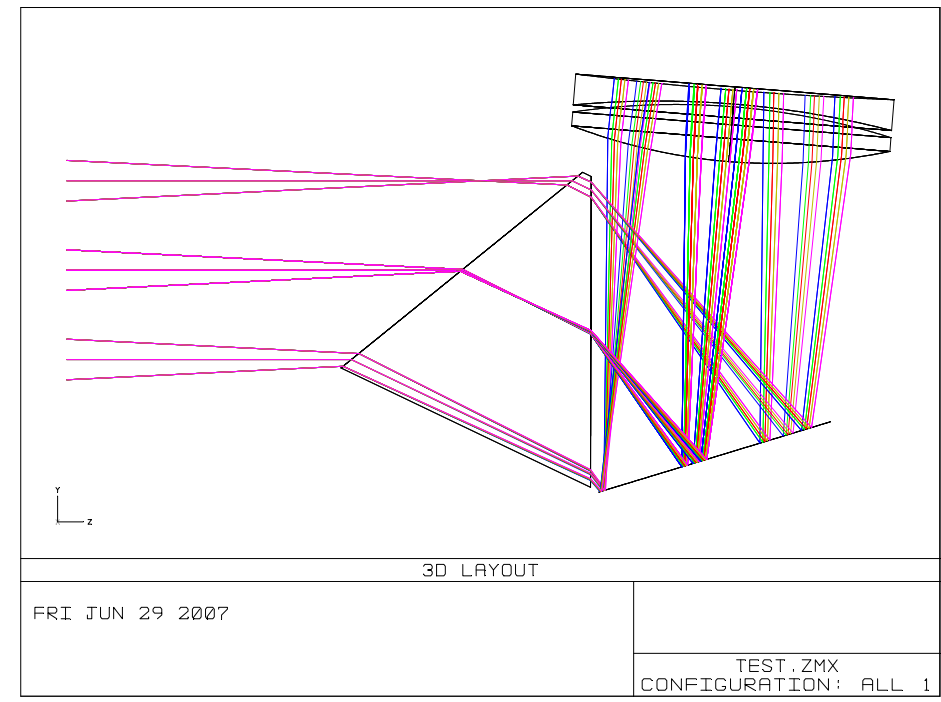

Figure 7. Prism, fold mirror and first 2 lenses of the 4-lenses camera.

The prism and his fold are replaced by a filter and another fold for imaging. Each filter has its own fold so that there is only one wheel per spectrograph for the prism, filters and fold mirrors.

\subsubsection{Camera}

The camera contains 4 lenses, 2 made of $\mathrm{CaF} 2$ and 2 of S-TIL26. The latter glass was chosen for his compatibility with $\mathrm{CaF} 2$ to remove the chromatic aberrations and for his low absorption. The CaF2 lenses each have an aspheric surface while all other surfaces of the camera are spherical. A fold mirror in the middle of the camera permits to place the detector near the telescope optical axis behind the pyramid mirror. This simplifies the detector cooling system since all 4 
detectors are near each other in this design. The average pan-chromatic PSF size between $0.8 \mu \mathrm{m}$ and $1.8 \mu \mathrm{m}$ is 0.07 ". This design could not be used for EUCLID because of the large number of optics.

\section{PRE-PHASE-A DESIGN OF THE EUCLID SPECTROGRAPH CHANNEL}

The EUCLID spectrograph channel designed by the first author of the present paper kept most of the characteristics of the SPACE design but with some significant differences ${ }^{5}$. This comes from 2 of the conditions that ESA placed on the design of EUCLID: (1) the DUNE telescope was to be used and the SPACE payload adapted to it; (2) the optical design of SPACE must be simplified to significantly reduce the number of optics. The DUNE telescope was a 1.2-m off-axis 3mirrors Korch design, not at all the 2-mirrors Ritchey-Chretien design of ATLAS. Different focal plane positions were studied for the pyramid mirror of the spectrograph channel. The intermediate focal plane between the secondary and tertiary seems the best. Its aberrations were corrected with the DMD fore-optics and the DUNE optics placed further out to leave some space for the spectrograph channel. The second condition forced a significant redesign of the fore-optics and spectrographs.

\subsection{Reduction of numbers of optics and detectors}

One of the conditions put forward by ESA was the need to reduce the number of optics in SPACE. Table 2 shows these reductions. The number of arms was reduced from 4 to 3 (fig. 8). However, since the telescope was reduced from $1.5-\mathrm{m}$ to $1.2-\mathrm{m}$, using the same spectrograph channel design would boost the micromirror size from 0.75 " to 0.94 ". The total field could then have been even larger in EUCLID even if the number of spectrographs was smaller. The spatial sampling would be worse however. A trade-off was made to get a resolution of 0.85 " and reduce the optics to fit behind the primary mirror of the telescope. The fore-optics were reduced from 4 to 3 mirrors; while this worsens the image quality, it is still at $3 \mu \mathrm{m}$ RMS radius much smaller than the micromirror.

The largest reduction was with the camera of the spectrograph (fig. 9). The 4 lenses and 2 folds were replaced by one concave mirror. To achieve the correct image quality, the perfect collimation on the prism had to be abandoned. This means that the collimator and camera optics work together to give the final image. They cancel each other's aberrations.

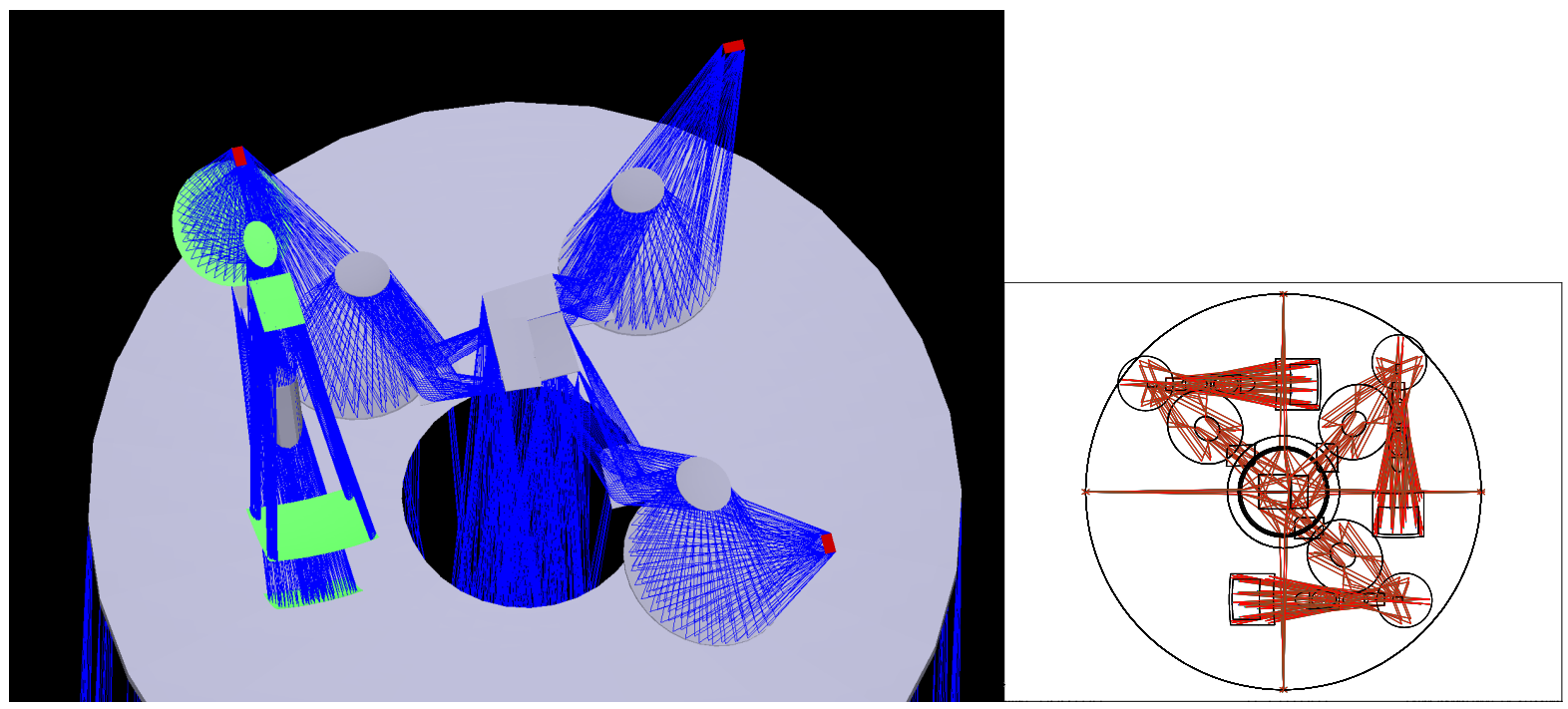

Figure 8. View of the EUCLID spectrograph channel with 2 of the 3 spectrographs removed.

This however makes it impossible to use the simple method of replacing the prism by a fold for imaging. With the beam uncollimated on the prism, a fold leaves large aberrations on the detector. A new imaging method had to be found. It had to more or less simulate the prism but without the dispersion. The solution to this problem was to use an achromatic 
grism with a shape almost identical to the prism using a glass with a lower dispersion than the prism glass; the grating and prism dispersions cancel each other (fig. 10). An excellent image quality was obtained.

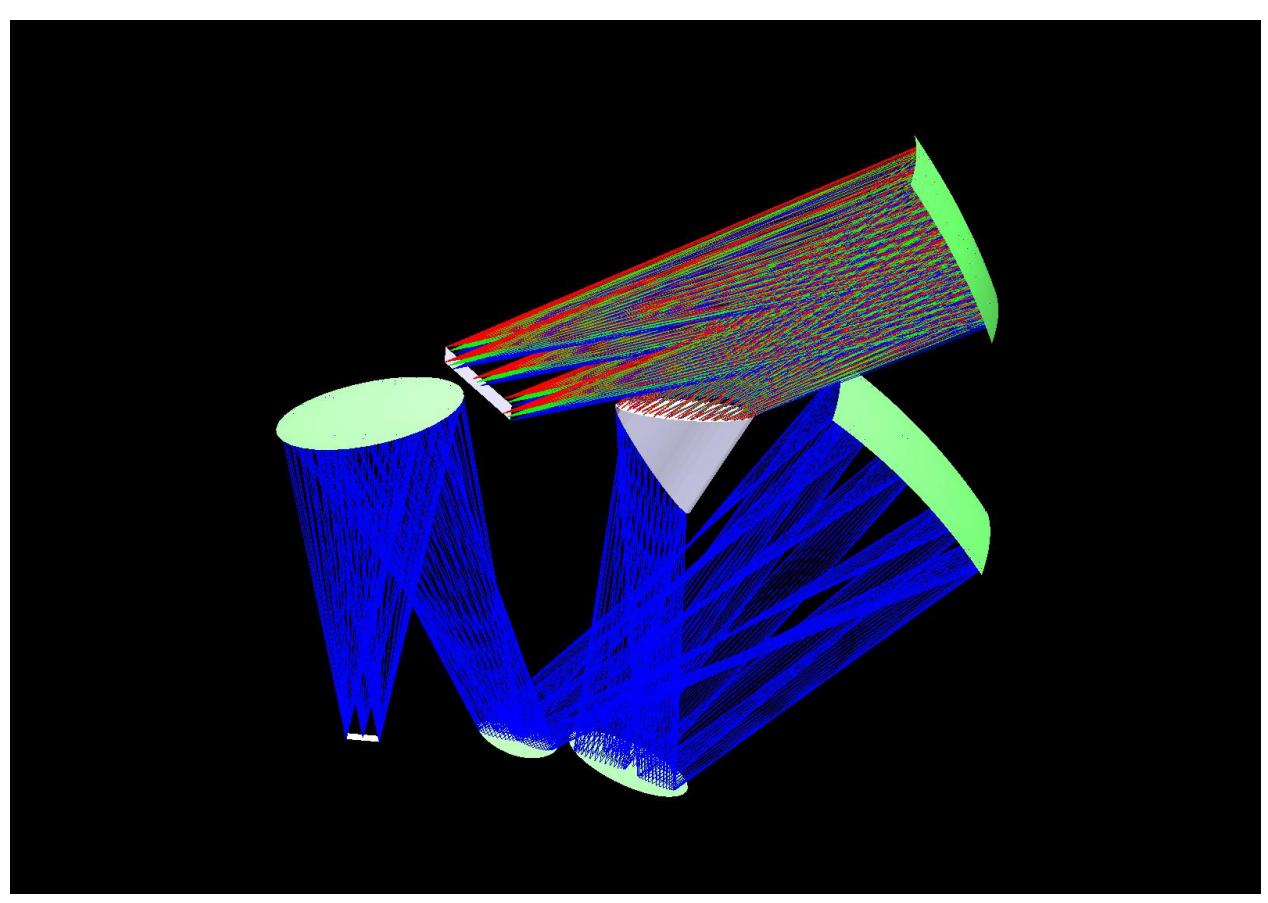

Figure 9. View of a spectrograph of the EUCLID spectrograph channel.

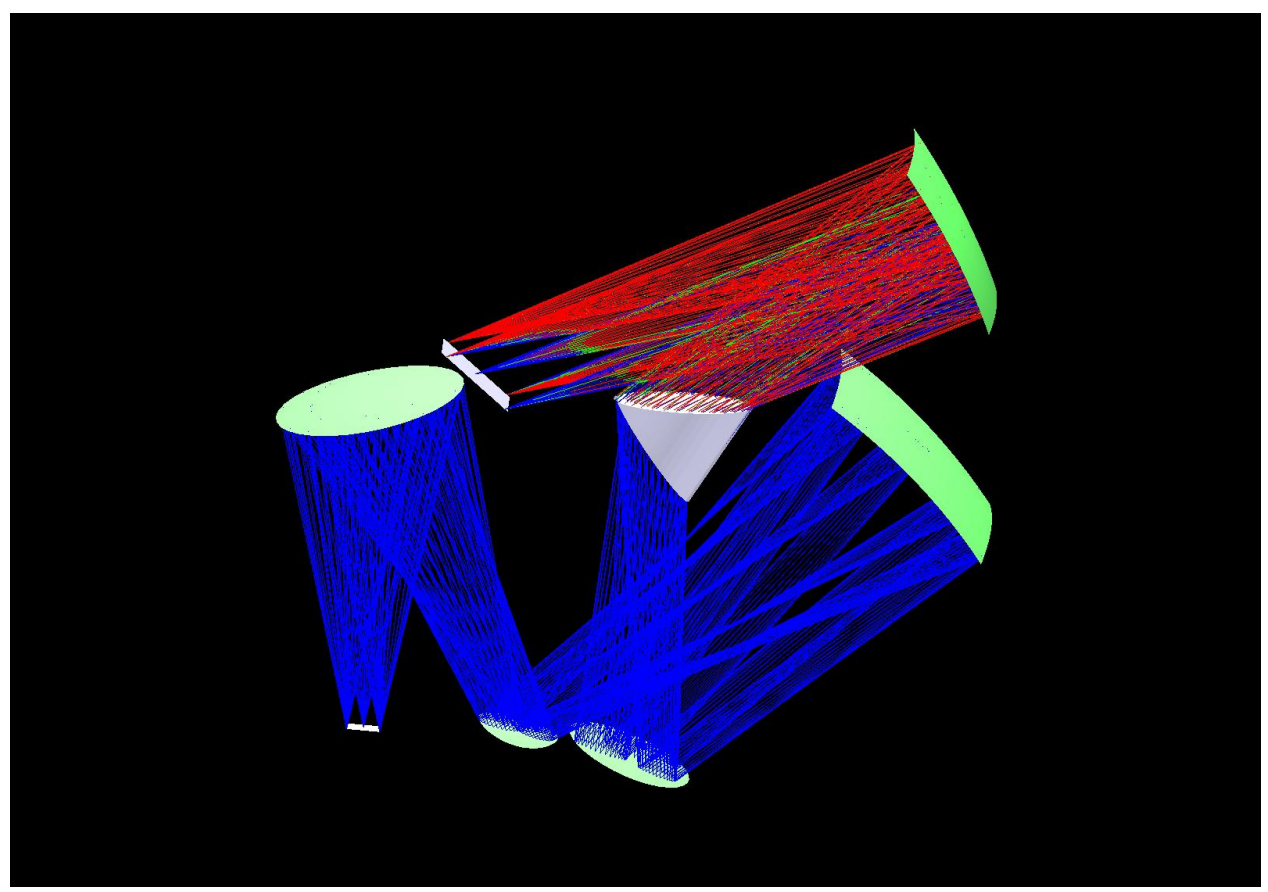

Figure 10. View of a spectrograph in imaging mode; while some dispersion is visible after the prism, the light comes together on the detector. 
All of the mirrors of the fore-optics and spectrographs were complex aspheres that were on the limit of what could be manufactured 10 years ago. Optics manufacturing has however progressed considerably since then and these aspheres can now easily be manufactured.

Table 2. Reductions in the EUCLID spectrograph channel compared to SPACE.

\begin{tabular}{|c|c|c|}
\hline $\begin{array}{c}\text { Number of } \\
\text { arms }\end{array}$ & SPACE & EUCLID-spectro \\
\hline Fore-optics & 4 mirrors & 3 mirrors \\
\hline camera & $\begin{array}{c}4 \text { lenses } \\
+2 \text { folds }\end{array}$ & 1 mirror \\
\hline $\begin{array}{c}\text { Number of } \\
\text { detectors per } \\
\text { spectrograph }\end{array}$ & 4 & 2 \\
\hline
\end{tabular}

The detector number had also to be reduced. Instead of $4 \times 2 \mathrm{k} x 2 \mathrm{k}$ per spectrograph, we had to use $2 \times 2 \mathrm{k} x 2 \mathrm{k}$. This was in fact possible because of the inevitable minimum gap between the 2 detectors which increases the total length and the ability to use a magnification slightly shorter in the spectral direction than in the spatial. Still, the resulting design was under-sampled in both directions. This will not be a problem with ATLAS since 4k x 4k detectors will soon be available.

\section{ATLAS PROBE}

The present preliminary design of ATLAS Probe contains characteristics of both SPACE and the pre-phase-A EUCLID spectrograph channel. As in SPACE, the telescope is a $1.5-\mathrm{m}$, there are 4 arms so 4 DMDs with 2048 x 1080 micromirrors $0.75 "$ x 0.75 " in size, and the field is nearly $0.4 \mathrm{deg}^{2}$. The fore-optics are however similar to that of the first design of EUCLID, especially they have only 3 re-imaging mirrors. The spectrograph design is also that of the original EUCLID with only one optics, a concave mirror, in each camera. The largest difference is that there are now 2 cameras. A dichroic was then inserted in the beam before the prism and a second camera was added to the EUCLID design. Also, the longest wavelength is $4 \mu \mathrm{m}$ which significantly influence the choice of glass of the prisms. In the red camera, no unique glass could do the job. The present design uses a $\mathrm{ZnSe}$ and fused silica prism doublet with a void gap to permit coatings of the surfaces since the index difference is too large. Another difference is that the DMD has been space qualified to level TL5 and can be qualified to TL6 in 2 years. Finally, the detectors are $4 \mathrm{k} \times 4 \mathrm{k}$ instead of the $4 \times 2 \mathrm{k} \times 2 \mathrm{k}$ of SPACE and the $2 \times 2 \mathrm{k} \times 2 \mathrm{k}$ of EUCLID.

The ATLAS telescope is a $1.5-\mathrm{m} \mathrm{f} / 15.3$ modified Ritchey-Chrétien. The secondary being quite small, only $3 \%$ of the input beam is lost by the obscuration. The telescope focal plane falls on a four-faceted pyramid mirror, a solution adopted e.g. by the WFPC2 camera on the Hubble Space Telescope. A full view of the ATLAS Probe instrument is shown in Figure 11. The 4 faces of the pyramid mirror act as pickoff mirrors (Figure 12, left) sending the light to a foreoptics system. The 4-petals shape of the field allows for almost perfect tessellation of the sky, except for 2 small squares totaling only $0.07 \%$ of the area, due to the aspect ratio of the DMD which is different from the ideal 2:1 ratio (full field coverage will be achieved through dithering). Figure 13 shows how the observed field add up to cover almost all the sky. Our approach also permits the split of the instrument into 4 identical and separable blocks, which helps simplify the assembly, integration and testing.

The 3 mirrors fore-optics (Figure 12, right) receive the $f / 15.3$ telescope beam and release a $\sim \mathrm{f} / 2.4 \times \mathrm{f} / 2.2$ beam on the DMD, accommodating for the $12^{\circ}$ micro-mirror tilt along the diagonal. The DMD acts as an addressable multi-slit mask, reflecting to the spectrograph the light of the science targets $\left(+12^{\circ}\right.$, ON beam $)$ and sending the rest of the field $\left(-12^{\circ}\right.$, OFF beam) to a light dump as in the SPACE and original EUCLID designs. 


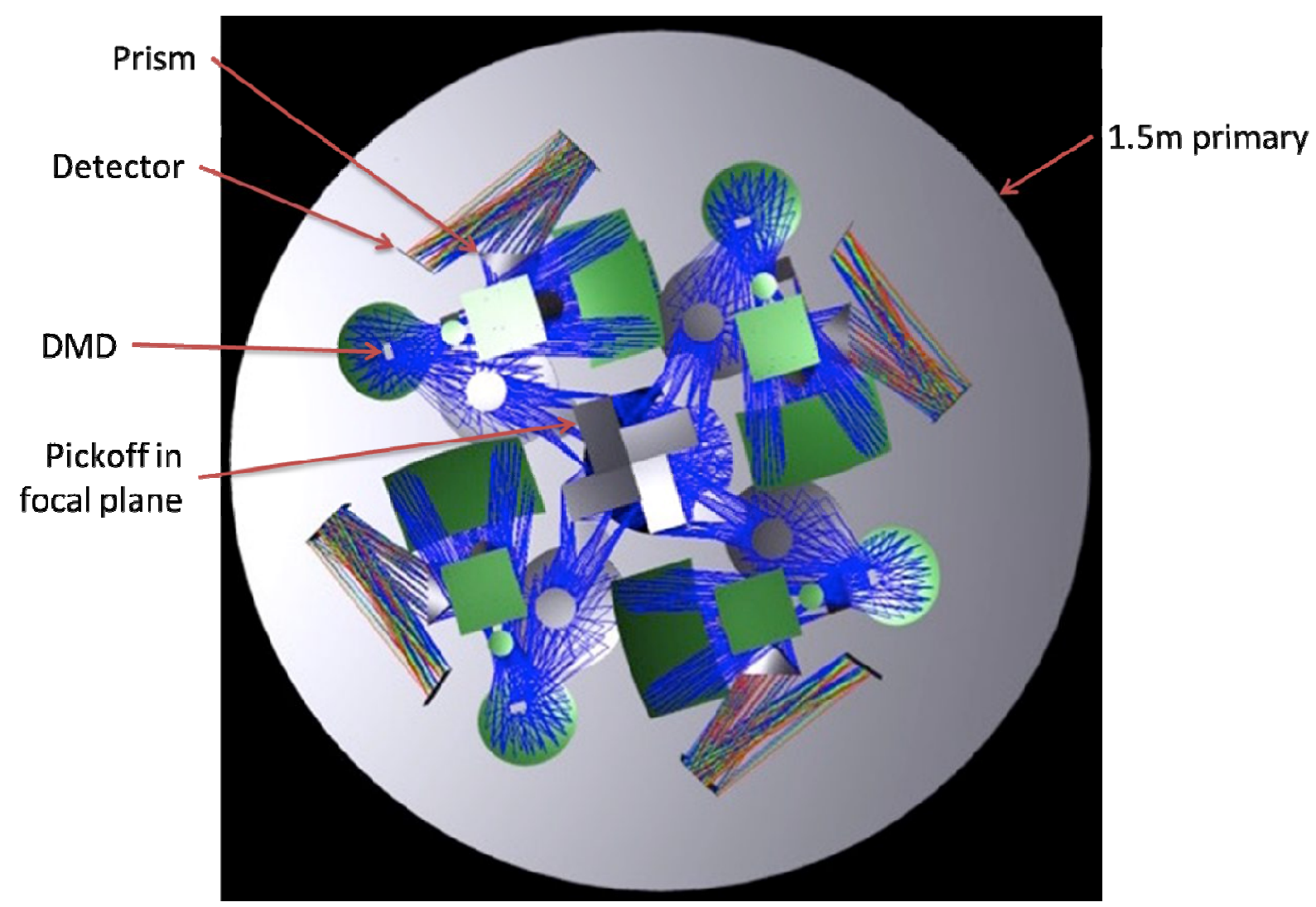

Figure 11. A full view of the preliminary optical design of the ATLAS Probe instrument. The large gray circle is the back of the primary.
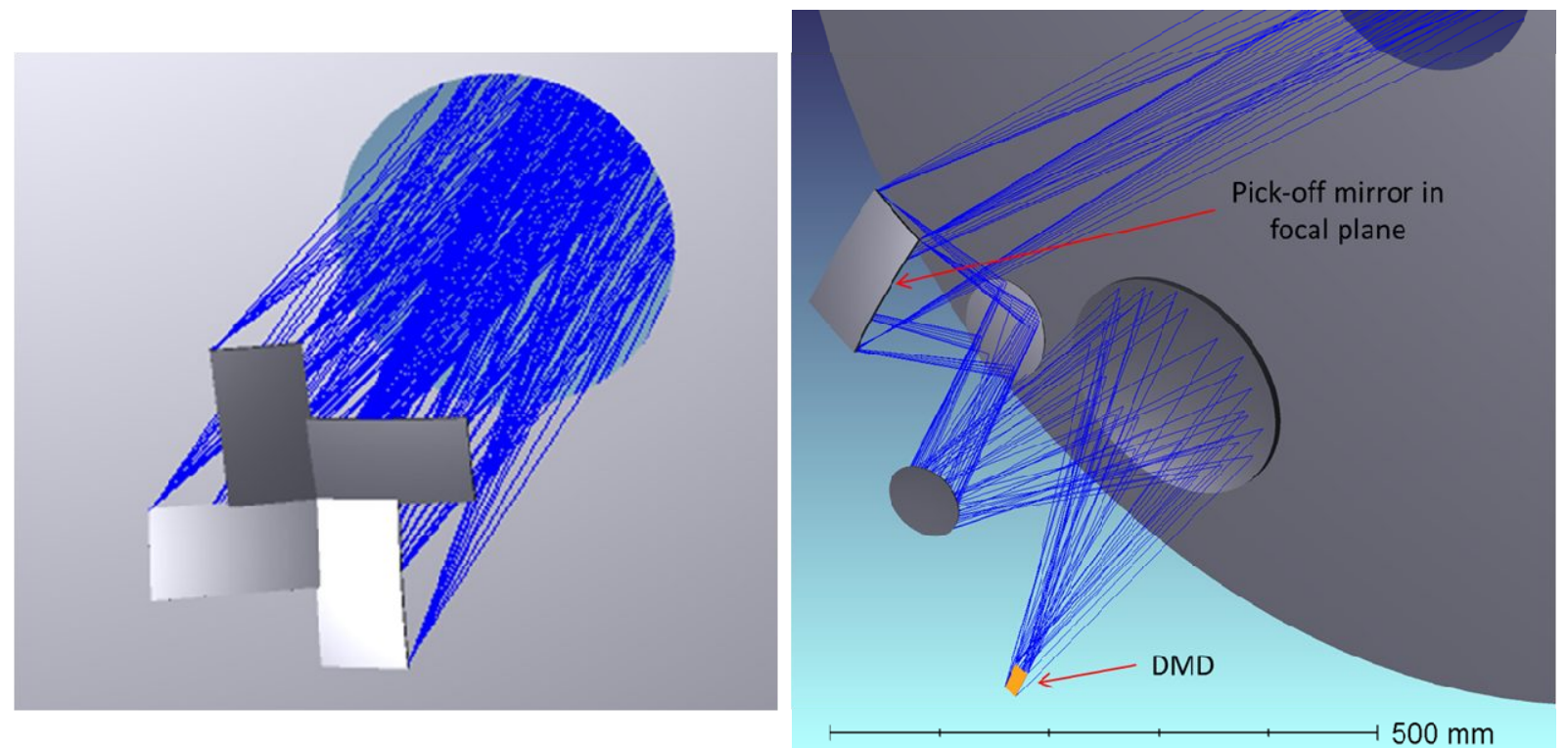

Figure 12. Preliminary optical design for the ATLAS Probe instrument. Left panel: Pickoff mirrors in focal plane; positions and shapes are approximate. Right panel: Shade layout of the fore-optics. 


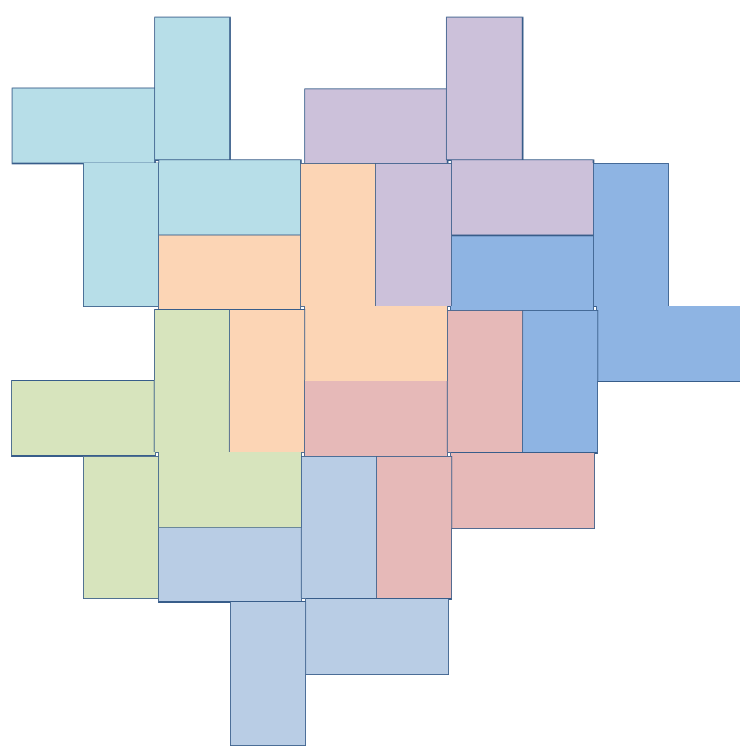

Figure 13. Tiling of the sky with a 4-petals pickoff if each petal has an aspect ratio of 1.896. The loss of coverage is only $0.07 \%$ apparent as small squares at half the corners of the petals.

The spectrograph (Figure 14) includes a 4 mirror collimator followed by a beam splitter reflecting and transmitting the beam to a short (1-2.1 $\mu \mathrm{m})$ and long-wavelength $(2.1-4 \mu \mathrm{m})$ channel, respectively. Similar beam splitters (with cut-off at 2.4 micron) have been adopted e.g. by NIRCam on JWST. We take advantage of the relatively modest resolving power $\mathrm{R}=600$ to make use of prisms instead of gratings as dispersive element. Prisms provide a series of advantages over gratings:

(1) They have a much better transmission especially at the extreme wavelengths of the spectra.

(2) They can have a wavelength range larger than an octave between the extreme wavelengths. In this preliminary design the short wavelength camera has a wavelength range from $1 \mu \mathrm{m}$ to $2.1 \mu \mathrm{m}$, with the possibility of further increasing the total wavelength range up to $0.9 \mu \mathrm{m}$ to $5 \mu \mathrm{m}$ in a future trade-off study.

(3) The resolution is more uniform as a function of wavelength.

(4) There is no need for a sorting order filter since there are no orders.

For target acquisition it is necessary to directly image the field; as originally proposed for EUCLID, direct imaging can be achieved through an achromatic grism where grating and prism dispersions cancel each other, with excellent image quality. ATLAS has 2 cameras, but only one is needed for target acquisition. Since the ATLAS short wavelength camera has a wavelength range only slightly different from EUCLID, we envision a slightly modified design of the EUCLID achromatic grism.

Our preliminary design already has excellent image quality in the spectrograph. The Gaussian Equivalent Full Width at Half Maximum (GEFWHM) is about 1/3 of a DMD mirror image. The image quality of the fore-optics on the DMD is $3 / 4$ of a DMD mirror, satisfactory at this point of the preliminary design, especially that it is necessary to well cover each micromirror to have a correct resolution on the detector and to avoid losses of light and calibration problems due to the small hole in the centre of each micromirror.

To attain the performances described above we make use of aspheric surfaces, some of them quite complex but still well inside the limits of modern manufacturing. Table 1 lists the main parameters of our system. Figure 15 shows the focal plane layout, with the DMD reimaged onto the lower part of the detector and the detector's upper part left "dark" to capture the full spectrum of each source, regardless of its position in the reimaged field. 


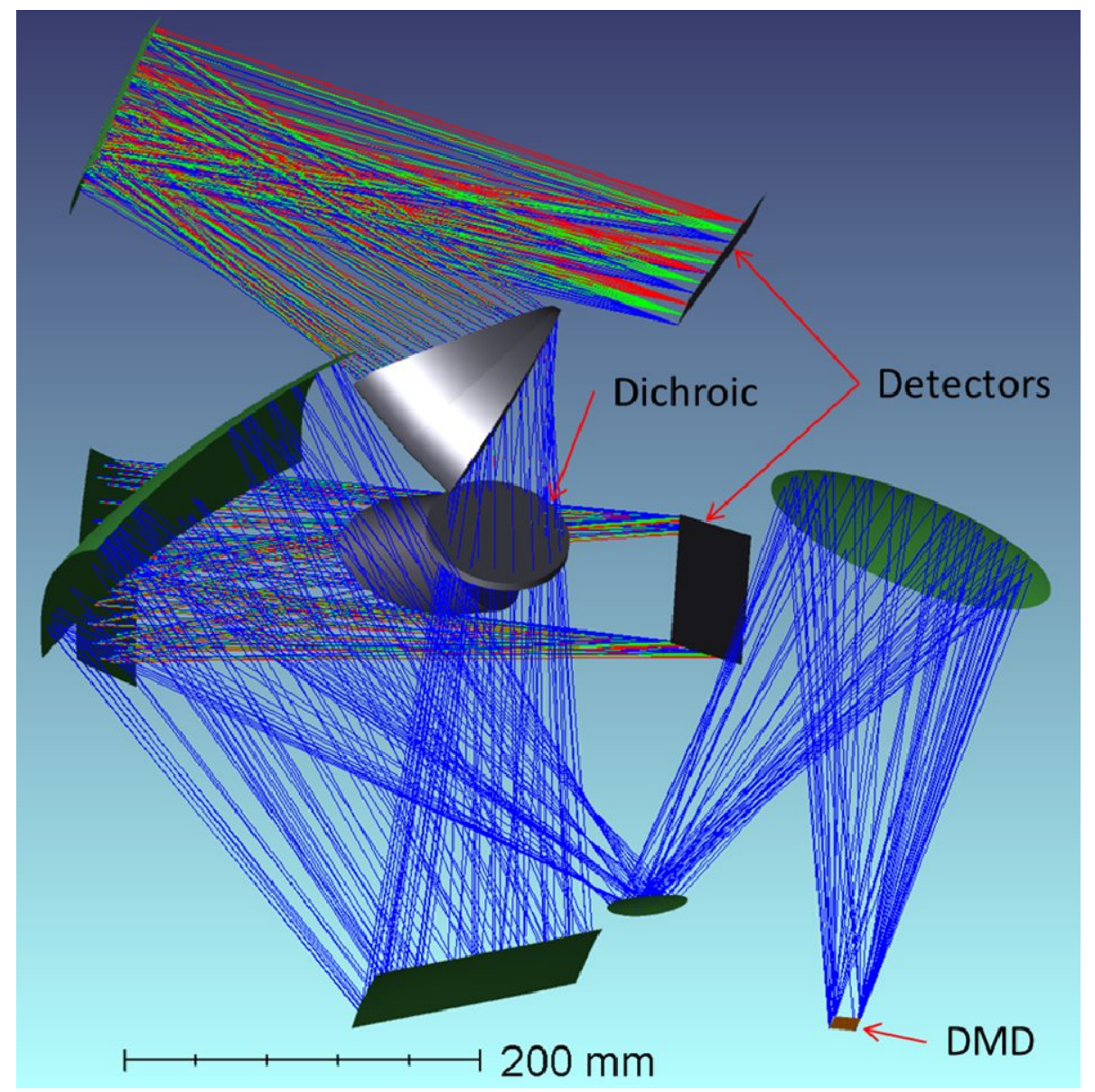

Figure 14. Preliminary optical design for the ATLAS Probe instrument. Shade layout of the spectrograph; the short wavelength camera is on the opposite side.

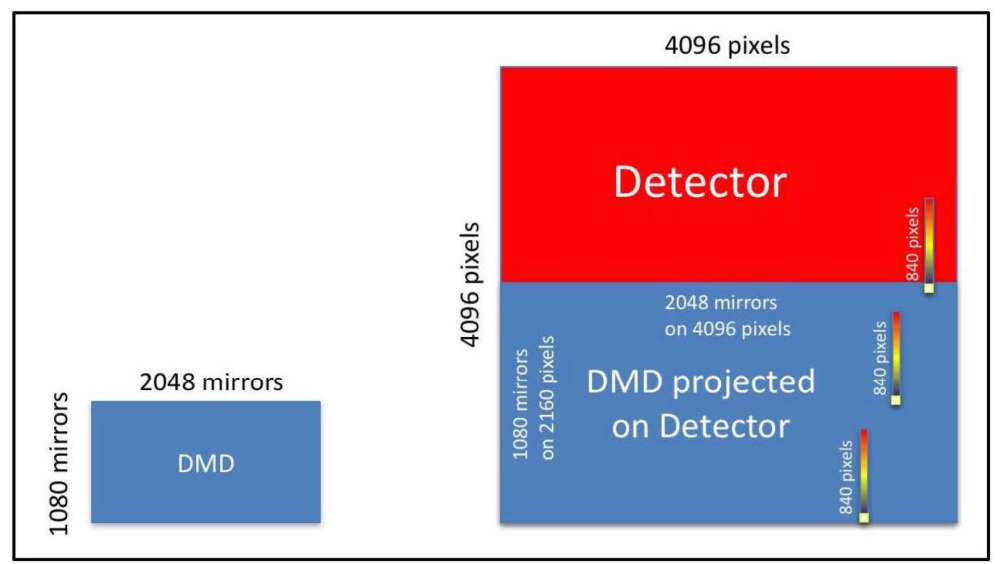

Figure 15. Layout of the focal planes for ATLAS Probe: left) focal plane projected on the DMD, right) focal plane project on the IR detector. The DMD is reimaged onto the lower part of the detector, and the detector's upper part is left "dark" to capture the full spectrum of each source, regardless of its position in the reimaged field. The moderate lengths of the $\mathrm{R} \sim$ 600 spectra allows for stacking multiple spectra on the same column, increasing source multiplexing. 


\section{CONCLUSION}

ATLAS Probe is a compelling mission concept for a NASA probe-class mission. It is a follow-up space mission to WFIRST; it multiplexes the scientific return of WFIRST by obtaining spectra of $\sim 90 \%$ of all galaxies imaged by the WFIRST High Latitude Survey at $\mathrm{z}>0.5$. Enabled by the mature DMD technology that allows a spectroscopic multiplex factor of 5,000 to 10,000, ATLAS Probe will lead to ground-breaking science over the entire range of astrophysics: from

galaxy evolution to the dark Universe, from objects in the outer Solar System to the dusty regions of the Milky Way.

In future work, we will mature the ATLAS Probe mission design and further quantify the science return from ATLAS Probe in its diverse science areas. In addition, we will engage the astronomical community in exploring feasible guest observing programs for ATLAS Probe.

\section{REFERENCES}

[1] Laureijs, R., et al, "Euclid Definition Study Report," arXiv:1110.3193 (2011).

[2] Spergel, D.N., et al, "WFIRST SDT Final Report," arXiv:1503.03757 (2015).

[3] Abell, P.A., et al, "LSST Science Book," arXiv:0912.0201 (2009).

[4] Cimatti, A., Robberto, M., et al (the SPACE Team), "SPACE: the SPectroscopic All-sky Cosmic Explorer," Exp. Astron., 23, 39-66 (2009).

[5] Content, R., "Durham design of EUCLID," Proc. SPIE 7010, 701025 (2008)

[6] Wang, Y., et al, "ATLAS Probe: breakthrough science of galaxy evolution, cosmology, Milky Way, and the solar system," arXiv:1802.01539 (2018)

[7] Wang, Y., Chuang, C.-H., Hirata, C.M., "Towards more realistic forecasting of dark energy constraints from galaxy redshift surveys," MNRAS, 430, 2446-2453 (2013).

[8] Aghamousa, A., et al, "The DESI Experiment Part I: Science,Targeting, and Survey Design," arXiv:1611.00036 (2016).

[9] Fraser, W. C., Brown, M. E., Glass, F., "The Hubble Wide Field Camera 3 Test of Surfaces in the Outer Solar System: Spectral Variation on Kuiper Belt Objects," ApJ, 804, 31 (2015).

[10] Refregier, A., Douspis, M., "Summary of the DUNE mission concept," Proc. SPIE 7010, 701018 (2008).

[11] Jeffrey T. Booth, J.T., Cropper, M.S., Eisenhauer, F., Refregier, A., "The focal plane instrumentation for the DUNE mission", Proc. SPIE 7010, 70101D (2008).

[12] Content, R., et al, "Offspring of SPACE: the Spectrograph Channel of the ESA Dark Energy Mission EUCLID”, Proc. SPIE 7010, 70104S (2008).

[13] Spanò, P., et al, "DMD multi-object spectroscopy in space: the EUCLID study," Proc. SPIE 7436, 74360O (2009). 